\title{
Optimization and Characterization of Artesunate-Loaded Chitosan-Decorated Poly(D,L-lactide-co-glycolide) Acid Nanoparticles
}

\author{
Hoang Nhan Ho, ${ }^{1,2}$ Tuan Hiep Tran, ${ }^{3}$ Trong Bien Tran, ${ }^{1}$ \\ Chul Soon Yong, ${ }^{3}$ and Chien Ngoc Nguyen ${ }^{1}$ \\ ${ }^{1}$ National Institute of Pharmaceutical Technology, Hanoi University of Pharmacy, 13-15 Le Thanh Tong, Hoan Kiem, \\ Ha Noi 100000, Vietnam \\ ${ }^{2}$ Hue College of Medicine and Pharmacy, Hue University, 6 Ngo Quyen, Hue, Thua Thien Hue 530000, Vietnam \\ ${ }^{3}$ College of Pharmacy, Yeungnam University, 214-1 Dae-Dong, Gyeongsan 712-749, Republic of Korea
}

Correspondence should be addressed to Chul Soon Yong; csyong@yu.ac.kr and Chien Ngoc Nguyen; nguyenngocchien@yahoo.com

Received 4 September 2015; Accepted 3 November 2015

Academic Editor: Ilaria Armentano

Copyright (C) 2015 Hoang Nhan Ho et al. This is an open access article distributed under the Creative Commons Attribution License, which permits unrestricted use, distribution, and reproduction in any medium, provided the original work is properly cited.

\begin{abstract}
The aim of this study was to optimize the formulation of artesunate-loaded chitosan- (CS-) decorated poly(D,L-lactide-coglycolide) acid (PLGA) nanoparticles as well as evaluate their characteristics. CS-to-PLGA mass ratio, $\mathrm{pH}$ of CS solution, and experimental temperature were optimized using response surface methodology to understand their effects on size and zeta potential of nanoparticles. The optimized formulation showed the close agreement between predicted and experimental values (all bias below $5 \%$ ). The presence of CS was confirmed by positive surface charge and Fourier transform infrared spectroscopy. A spherical-like shape of particles was observed in range of small size around $190 \mathrm{~nm}$. This CS layer restricted initial burst release of drug from carriers in phosphate buffer of $\mathrm{pH}$ 6.8. In addition, CS-coated NPs enhanced the intracellular uptake, in vitro cytotoxicity, and apoptosis-induced nuclei behaviors compared with CS-uncoated NPs as well as free drug in MCF-7 and A549 cancer cells.
\end{abstract}

\section{Introduction}

Artesunate (ART) is a semisynthetic derivative of artemisinin, which is the active principle of the traditional herb Artemisia annua, and is one of potential antimalarial treatments [1]. Due to its strong cytotoxicity, ART has recently been the subject for various studies about its effects on cancer cell lines. It is shown that ART plays an important role against leukemia, melanoma, non-small-cell lung cancer, colon cancer, renal cancer, ovarian cancer, prostate cancer, central nervous system cancer, and breast cancer $[2,3]$. Several antitumoral mechanisms of ART are studied such as modulating genes and proteins, coordinating growth signals, apoptosis, proliferation capacity, angiogenesis and tissue invasion, and metastasis, for example, p53, Bcl-2 familymediated mitochondrial dysfunction, and enhanced reactive oxygen species (ROS) production $[1,2]$. However, ART is not very stable, most probably by the opening of the lactone ring, due to its unusual peroxy group and poor aqueous solubility [4]. Therefore, development of a drug delivery carrier that can maintain sustained release profile and avoid rapid degradation is essential for effective therapy of ART [5].

Poly(D,L-lactide-co-glycolide) acid (PLGA) is the widely used biodegradable and biocompatible polymer that has been approved by the FDA for drug delivery systems (DDS) [6, 7]. Furthermore, PLGA-based DDS can provide a sustained and controlled drug release and reduce side effects. In the previous study, PLGA-based NPs were fabricated containing 
ART using oil/water emulsion evaporation method. The results showed particle size of $170 \mathrm{~nm}$, high entrapment efficiency (up to $83.4 \%$ ), and good stability (evaluated for 1 month) after lyophilization with $5 \%$ of mannitol. ART was dispersed inside particle core allowing a sustained release up to $48 \mathrm{~h}$. The ART-PLGA formulation significantly reduced cell viability compared to the free ART in the in vitro cytotoxicity test with three cancer cell lines such as A549, SCC-7, and MCF-7 by using MTT assay [8]. However, the PLGA NPs have low intracellular uptake because the slightly negative surface charge of PLGA nanoparticles (PLGA with carboxylic end-group) tends to limit their interaction with the negatively charged cell surface, especially of cancer cells. In addition, due to a deficiency of functional groups on PLGA surface, these DDS are believed to be difficult to achieve functionalities such as passive targeting and active targeting [9].

In current study, a positively charged polymer, chitosan, was decorated on the surface of PLGA-based NPs in order to enhance intracellular uptake of NPs. For the first time, experiment design was used to optimize as well as predict properties of chitosan-coated PLGA NPs using MODDE software. The change of physicochemical characteristics such as surface charge, surface properties, and particle size was evaluated by dynamic light scattering (DLS), Fourier transform infrared (FT-IR) spectroscopy, and transmission electron microscopy (TEM). Furthermore, the superiority of chitosan-coated PLGA NPs was determined on various cancer cell-lines: MCF-7, A549 in case of in vitro cytotoxicity and intracellular uptake as well as nuclei behaviors.

\section{Materials and Methods}

2.1. Materials. Artesunate (ART) was provided by Sao Kim Pharma (Hanoi, Vietnam). PLGA (Lakeshore 5050 DLG 2A) was purchased from Evonik (Kirschenallee, Darmstadt, Germany). Chitosan (low molecular weight, 50-190 kDa, 75-85\% deacetylated), a cationic polysaccharide, was purchased from Sigma-Aldrich Chemical Co. Ltd., Sigma (St. Louis, MO, USA). Polysorbate 80 (Tween 80 ) was purchased from Duksan Chemical Co. (Ansan, Korea). Sucrose, trehalose, mannitol, DMSO for cell culture, and Thiazolyl Blue Tetrazolium Bromide (MTT) were purchased from Sigma-Aldrich Chemical Co. Ltd., Sigma (St. Louis, MO, USA), whereas 2,5' -Bi1H-benzimidazole, $2^{\prime}$-(4-ethoxyphenyl)-5-(4-methyl-1-piperazinyl)-23491-52-3 (Hoechst 33342) was supplied by Thermo Fisher Scientific Inc. (Waltham, MA, USA). Methanol, acetonitrile, and dimethyl sulfoxide (DMSO) were of HPLC grade. All other chemicals were of analytical grade and were used without further purification.

A549 (human lung adenocarcinoma) and MCF-7 (human breast cancer) were obtained from Korean cell bank and cultured in Dulbecco's Modified Eagle Medium (DMEM), high glucose (HyClone Lab., South Logan, Utah, USA) supplemented with $10 \%$ fetal bovine serum (FBS) and $1 \%$ penicillin/streptomycin, in a humidified atmosphere containing $5 \% \mathrm{CO}_{2}$ at $37^{\circ} \mathrm{C}$.

\subsection{Methods}

2.2.1. Preparation of PLGA-CS Nanoparticles. PLGA nanoparticles containing ART were prepared using a single emulsion solvent evaporation method with slight modifications as previously reported [8]. Briefly, $20 \mathrm{mg}$ of ART and $50 \mathrm{mg}$ of PLGA were dissolved in $5 \mathrm{~mL}$ dichloromethane (DCM) and this solution was added drop-wise to $50 \mathrm{~mL}$ of aqueous solution containing $1.5 \%(\mathrm{w} / \mathrm{v})$ emulsifier Tween 80 . The oilin-water emulsion was fabricated by homogenization using high-intensity probe sonicator, Vibracell VCX130 (Sonics \& Materials, Newtown, CT, USA) at $100 \mathrm{~W}$ for $5 \mathrm{~min}$ in ice cold water $\left(5-10^{\circ} \mathrm{C}\right)$. The emulsion formed was stirred for $4 \mathrm{~h}$ at $1,000 \mathrm{rpm}$ at room temperature to evaporate the organic solvent. The coumarin 6-loaded nanoparticles for the intracellular uptake study were prepared under the same conditions as above except that ART was replaced by coumarin 6. To prepare CS-modified PLGA nanoparticles, PLGA nanoparticles were added to the chitosan solution in $1 \%(\mathrm{v} / \mathrm{v})$ acetic acid at different $\mathrm{pH}$ (adjusted by $3 \mathrm{~N} \mathrm{NaOH}$ solution), under agitation with a magnetic stirrer [10]. The nanosuspension was centrifuged at $12,000 \mathrm{rpm}$ for $30 \mathrm{~min}$ at $4^{\circ} \mathrm{C}$ (Centrifuge $5415 \mathrm{R}$, Eppendorf, Germany) and then resuspended in water. Dry powder was obtained by freeze drying (FDA5518, ILSHIN Biobase Co., Ltd., South Korea) in presence of cryoprotectants.

2.2.2. Freeze-Thaw Study. Freeze-thaw study was carried out in triplicate by freezing ART-loaded PLGA at $-70^{\circ} \mathrm{C}$ for $24 \mathrm{~h}$ followed by thawing at $25^{\circ} \mathrm{C}$ with the presence of $5 \%(\mathrm{w} / \mathrm{v})$ of dextrose, trehalose, sucrose, and mannitol. The particle size and PDI before freezing and after thawing were determined by DLS, and then the final size $\left(S_{f}\right)$ and initial size $\left(S_{i}\right)$ ratio was calculated [11].

2.2.3. Lyophilization. The dispersion was prefrozen $\left(-70^{\circ} \mathrm{C}\right)$ for $12 \mathrm{~h}$ and subsequently lyophilized at a temperature of $-25^{\circ} \mathrm{C}$ for $24 \mathrm{~h}$ followed by a secondary drying phase of $12 \mathrm{~h}$ at $20^{\circ} \mathrm{C}[8]$.

2.2.4. Experimental Design. Response surface methodology (RSM) is a statistical and mathematical approach for fitting the experimental data to a model for optimization. In this study, the relationships between independent variables and their responses were evaluated in an effective model using Doptimal experimental design (DOD) [12-14].

Three levels for each of the investigated factors were selected to generate the RSM design, the D-optimal. The design matrix comprised of 18 experimental runs for the DOD using MODDE 8.0 software along with contour surface response was used to optimize the formulation of ARTloaded PLGA-CS NPs. The CS-to-PLGA mass ratio $\left(X_{1}\right) ; \mathrm{pH}$ of CS solution $\left(X_{2}\right)$; and experimental temperature $\left(X_{3}\right)$ were the three independent factors. The dependent variables were the size $\left(Y_{1}\right)$, PDI $\left(Y_{2}\right)$, and zeta potential $\left(Y_{3}\right)$ of these NPs.

A second-order polynomial function model was employed to find the mathematical relationship between responses $\left(Y_{i}\right.$, size, PDI, and zeta potential) and the independent 
variables $\left(X_{i}\right.$, CS-to-PLGA mass ratio, $\mathrm{pH}$ of CS solution, and experimental temperature):

$$
\begin{aligned}
Y_{i}= & b_{0}+b_{1} X_{1}+b_{2} X_{2}+b_{3} X_{3}+b_{4} X_{1} X_{1}+b_{5} X_{2} X_{2} \\
& +b_{6} X_{3} X_{3}+b_{7} X_{1} X_{2}+b_{8} X_{1} X_{3}+b_{9} X_{2} X_{3},
\end{aligned}
$$

where $Y_{i}$ is the predicted response; $b_{0}$ is intercept; $b_{1}$ to $b_{9}$ are regression coefficients of the equation; and $X_{1}, X_{2}$, and $X_{3}$ are the independent variables.

\subsubsection{Measurement of Particle Size and Morphology. Particle} size and size distribution of nanoparticles were measured by the dynamic light scattering (DLS) method using Zetasizer Nano 90 (Malvern Instruments Ltd., Worcestershire, UK). All the samples for the analysis were prepared by redispersing nanoparticles in distilled water. The intensity of scattered light was detected at $90^{\circ}$ to an incident. All the data analysis was performed in automatic mode. Measured size was presented from the average value of 20 runs, with triplicate measurements within each sample.

The morphology of nanoparticles was evaluated by using a transmission electron microscope (TEM H7600, Hitachi, Tokyo, Japan) at an accelerating voltage of $100 \mathrm{kV}$. ARTloaded PLGA-CS nanoparticles were placed on a copper grid coated with a carbon film. Negative staining was performed by using phosphotungstic acid $(2 \%, \mathrm{w} / \mathrm{v})$ and then the grid was dried at room temperature prior to observation [15].

2.2.6. FT-IR Analysis. The surface chemistry and physical interaction between polymers and ART were determined from FT-IR spectra. Samples for FT-IR spectrometry were analyzed over the $550-4,000 \mathrm{~cm}^{-1}$ range using a Thermo Scientific Nicolet Nexus 670 FT-IR Spectrometer and Smart iTR software with a diamond window (Thermo Fisher Scientific Inc., Waltham, MA, USA) $[9,16]$.

2.2.7. Loading Capacity and Encapsulation Efficiency. To determine the total drug content, a suspension of ART-loaded PLGA-CS nanoparticles $(1 \mathrm{~mL})$ was placed in an ultrafiltration tube (MWCO 10000, Millipore, Billerica, MA, USA) and was centrifuged at 4,500 rpm for $15 \mathrm{~min}$ [8]. Then the filtrate was analyzed for free drug using HPLC. Encapsulation efficiency (EE) and loading capacity (LC) were calculated using equations as follows:

$$
\begin{aligned}
\mathrm{EE}(\%)= & \frac{\text { Total ART }- \text { Free ART }}{\text { Total ART }} \times 100 \%, \\
\mathrm{LC}(\%)= & \frac{\text { Weight of ART in nanoparticles }}{\text { Weight of ART/PLGA }- \text { CS nanopartilces }} \\
& \times 100 \% .
\end{aligned}
$$

2.2.8. In Vitro Drug Release. Drug release studies were carried out in a dialysis bag (molecular weight cut-off $10 \mathrm{kDa}$, Membrane-Cell, Chicago, IL, USA) containing $3 \mathrm{~mL}$ of ART/PLGA-CS suspension. The dialysis bag was placed in a $50 \mathrm{~mL}$ tube containing $10 \mathrm{~mL}$ of phosphate buffer $\mathrm{pH}$
6.8 (PBS) as release media. The tube was capped and placed on a shaking water bath (HST-205 SW, Hanbaek ST Co., Gyeonggi, Korea) rotating at $100 \mathrm{rpm}$ and maintained at $37^{\circ} \mathrm{C}$. At predetermined time points, $1 \mathrm{~mL}$ of sample was collected and replaced with fresh media after sampling. The quantity of ART was measured by Hitachi HPLC system consisting of a pump (Model L2100), an autosampler (Model L2200), an ultraviolet detector (Model L2420), and C18 analytic column (Inertsil ODS3: $15 \mathrm{~cm} \times 0.46 \mathrm{~cm}, 5 \mu \mathrm{m}$, GL Sciences Inc., Tokyo, Japan). The mobile phase was the mixture of acetonitrile and phosphate buffer solution with $\mathrm{pH} 3.0$ (55:45, volume per volume ratio). The UV absorbance was measured at wavelength of $216 \mathrm{~nm}$ with $1.0 \mathrm{~mL} / \mathrm{min}$ flow rate and $50 \mu \mathrm{L}$ injection volume $[8,15]$.

2.2.9. Intracellular Uptake by Fluorescence Activated Cell Sorting (FACS). MCF-7 and A549 cells were seeded in 6well tissue culture plates, at a density of $2 \times 10^{5}$ cells/well, and grown in DMEM at $37^{\circ} \mathrm{C}, 5 \% \mathrm{CO}_{2}$ for $24 \mathrm{~h}$. The cells were washed by PBS and then $1 \mathrm{~mL}$ of suspension containing coumarin 6-loaded PLGA or PLGA-CS nanoparticles in DMEM at the concentration of 2 or $4 \mu \mathrm{g} / \mathrm{mL}$ of coumarin 6 was applied to each well, at $37^{\circ} \mathrm{C}$ for $30 \mathrm{~min}$ or $60 \mathrm{~min}$. Following the removal of the supernatants, the cells were trypsinized and collected into FACS tubes before being centrifuged at $1500 \times \mathrm{g}$ for $5 \mathrm{~min}$ to obtain cell pellets. The pellets were washed twice with ice-cold PBS and then resuspended in $1.5 \mathrm{~mL}$ of PBS. The fluorescence signal intensities from the samples were measured using a flow cytometer (BD FACS Verse, BD Biosciences, USA) [10, 17].

2.2.10. In Vitro Cytotoxicity of ART-Loaded PLGA-CS Nanoparticles. Cell proliferation was assessed by MTT assay. Briefly, $100 \mu \mathrm{L}$ of cell suspension at a density of $1 \times 10^{4}$ cells per $\mathrm{mL}$ was seeded into 96-well tissue culture plates and incubated for $24 \mathrm{~h}$. The samples of blank PLGA-CS, free ART, ART-PLGA, and ART/PLGA-CS (concentrations from 6.25 to $100 \mu \mathrm{g} / \mathrm{mL}$ of drug amount) were added and incubated for $24 \mathrm{~h}$. The media with the samples were removed and MTT solution ( $100 \mu \mathrm{L}$ of $1.25 \mathrm{mg} / \mathrm{mL}$ MTT in supplemented DMEM medium) was added to each well and then incubated for $4 \mathrm{~h}$ at $37^{\circ} \mathrm{C}$. The medium with MTT solution was discarded and formazan crystals were solubilized using $100 \mu \mathrm{L}$ of DMSO. Subsequently, an incubation of 15 min under light protected condition and at room temperature was performed. The absorbance was measured at $570 \mathrm{~nm}$ using a microplate reader (Multiskan EX, Thermo Scientific, Waltham, MA, USA). Cell viability was calculated by the following formula $[15,18]$ :

$$
\begin{aligned}
\text { Cell viability }(\%)= & \frac{\mathrm{OD}_{570}(\text { sample })-\mathrm{OD}_{570}(\text { blank })}{\mathrm{OD}_{570}(\text { control })-\mathrm{OD}_{570}(\text { blank })} \\
& \times 100 .
\end{aligned}
$$

2.2.11. Nuclei Morphological Characterization. MCF-7 and A549 cells were seeded in 6-well tissue culture plates, at a density of $2 \times 10^{5}$ cells/well, and grown in DMEM at $37^{\circ} \mathrm{C}, 5 \%$ $\mathrm{CO}_{2}$ for $24 \mathrm{~h}$. After being treated with free drug, PLGA NPs, 
TABLE 1: Formulations of ART-loaded PLGA-CS NPs and their physicochemical characteristics.

\begin{tabular}{|c|c|c|c|c|c|c|}
\hline Number & CS/PLGA ratio (w/w) & $\mathrm{pH}$ (CS solution) & Temp. $\left({ }^{\circ} \mathrm{C}\right)$ & Size $(\mathrm{nm})$ & PDI & Zeta potential $(\mathrm{mV})$ \\
\hline 1 & 1.00 & 3.0 & 10 & 252.0 & 0.287 & 56.9 \\
\hline 2 & 0.20 & 5.0 & 10 & 289.8 & 0.284 & 33.0 \\
\hline 3 & 1.00 & 5.0 & 10 & 278.5 & 0.22 & 33.9 \\
\hline 4 & 0.20 & 3.0 & 40 & 198.5 & 0.233 & 45.3 \\
\hline 5 & 1.00 & 3.0 & 40 & 272.2 & 0.299 & 64.9 \\
\hline 6 & 0.20 & 5.0 & 40 & 321.3 & 0.263 & 39.2 \\
\hline 7 & 1.00 & 5.0 & 40 & 317.4 & 0.263 & 38.6 \\
\hline 8 & 0.20 & 3.0 & 20 & 190.5 & 0.205 & 34.9 \\
\hline 9 & 0.20 & 3.0 & 30 & 189.5 & 0.228 & 28.3 \\
\hline 10 & 0.20 & 3.7 & 10 & 187.8 & 0.192 & 23.1 \\
\hline 11 & 0.20 & 4.3 & 10 & 246.8 & 0.231 & 31.4 \\
\hline 12 & 0.47 & 3.0 & 10 & 200.0 & 0.265 & 40.8 \\
\hline 13 & 1.00 & 4.0 & 25 & 237.2 & 0.264 & 30.9 \\
\hline 14 & 0.60 & 5.0 & 25 & 263.5 & 0.235 & 36.4 \\
\hline 15 & 0.60 & 4.0 & 40 & 253.6 & 0.247 & 38.8 \\
\hline 16 & 0.60 & 4.0 & 25 & 213.0 & 0.227 & 35.3 \\
\hline 17 & 0.60 & 4.0 & 25 & 212.1 & 0.219 & 31.5 \\
\hline 18 & 0.60 & 4.0 & 25 & 205.7 & 0.211 & 32.1 \\
\hline
\end{tabular}

PLGA-CS NPs for $24 \mathrm{~h}$, the cells were washed with PBS three times and fixed with $4 \%$ formaldehyde at room temperature for $10 \mathrm{~min}$. Then the cells were rinsed with PBS before being stained with $5 \mu \mathrm{g} / \mathrm{mL}$ of Hoechst 33342 at room temperature for $15 \mathrm{~min}$ in the dark. Cells were then washed three times with PBS. The images of cell nuclei were captured using a fluorescence microscopy (Nikon, Tokyo, Japan).

2.2.12. Statistical Analysis. All data are expressed as mean \pm standard deviation (SD) from three replicates. The significance of differences was assessed by analysis of variance (ANOVA) and considered statistically significant when $p<$ 0.05 in all cases.

\section{Results and Discussion}

Chitosan, a naturally occurring linear polysaccharide and amino polysaccharide ( $\operatorname{poly}(\beta-[1,4]-\mathrm{D}$-glucosamine $))$, seems to be the most suitable adjuvant due to its biodegradable and biocompatible, mucoadhesive, and permeability-enhancing properties. This property enables chitosan to have in vitro cytotoxicity against different kinds of human cancer cell lines [19]. In this study, chitosan was decorated on the PLGA NPs surface to enhance strength of carriers on treating cancer. Moreover, the desired particles were optimized and obtained by experiment design.

3.1. Preparation and Optimization of ART-Loaded PLGA-CS. A preliminary study was carried out to screen and select the input variables that influence the response variables designed
TABLE 2: Summary of statistical results from the final regression model and full model.

\begin{tabular}{lccc}
\hline Parameter & $Y_{1}$ & $Y_{2}$ & $Y_{3}$ \\
\hline$R^{2}$ & 0.969 & 0.779 & 0.901 \\
$R^{2}$ adjusted & 0.935 & 0.530 & 0.790 \\
$Q^{2}$ & 0.827 & 0.411 & 0.417 \\
$p$ value & $\mathbf{0 . 0 0 0}$ & $\mathbf{0 . 0 6 2}$ & $\mathbf{0 . 0 0 4}$ \\
$p$ value (lack of fit) & $\mathbf{0 . 0 9 3}$ & $\mathbf{0 . 1 0 8}$ & $\mathbf{0 . 1 4 3}$ \\
\hline
\end{tabular}

by Modde 8.0. Table 1 shows the 18 formulations of PLGACS NPs along with their morphological characteristics such as size, PDI, and zeta potential.

In our work, ART-loaded PLGA-CS nanoparticles were obtained with size, PDI, and zeta potential ranging from 187 to $321 \mathrm{~nm},<0.300$ and 23 to $65 \mathrm{mV}$, respectively (Table 1 ). In order to select the best models fitted with the data, the analysis of variances by calculating $F$-values was used. Therefore, for each of three responses, a quadratic second-order polynomial equation was matched to the data using the software. The statistical results of ANOVA for all the responses are shown in Table 2 including the multiple correlation coefficients $\left(R^{2}\right)$, adjusted multiple correlation coefficients ( $R^{2}$ adjusted), predicted multiple correlation coefficients $\left(Q^{2}\right)$, and the $p$ value of the final model of each response variable. The $R^{2}$ and $R^{2}$ adjusted values were higher at size $\left(R^{2}\right.$ and $R^{2}$ adjusted > 0.9). $Q^{2}$ shows how well the model predicts new data; the higher the value of $Q^{2}$, the better the data prediction [14]. 


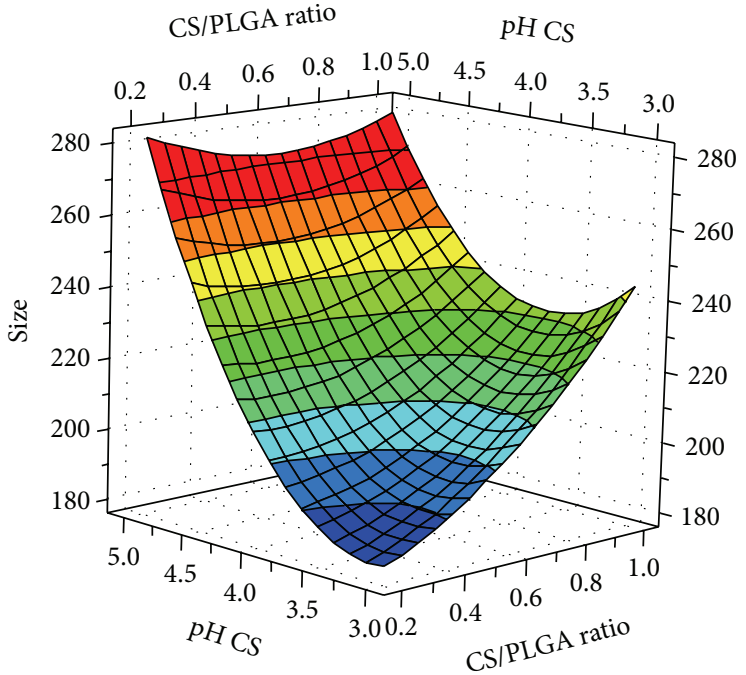

(a)

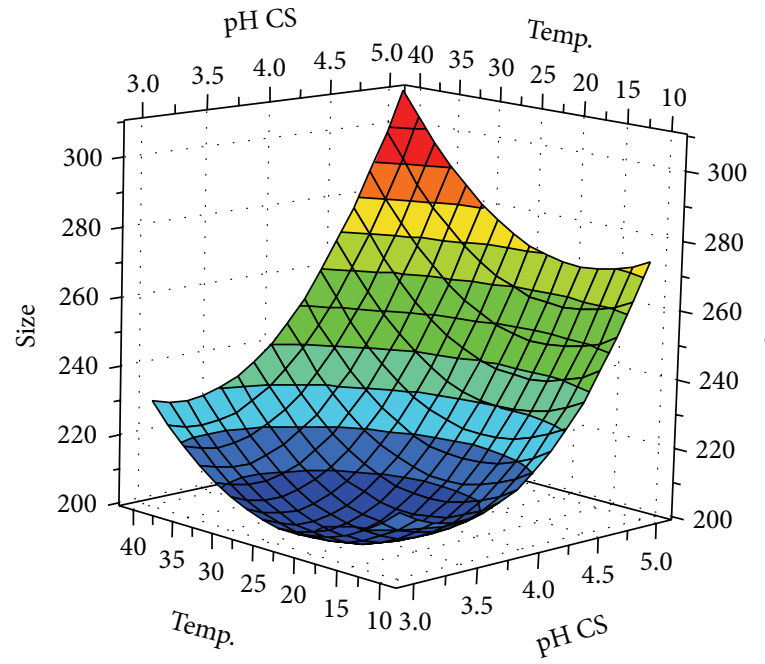

(c)

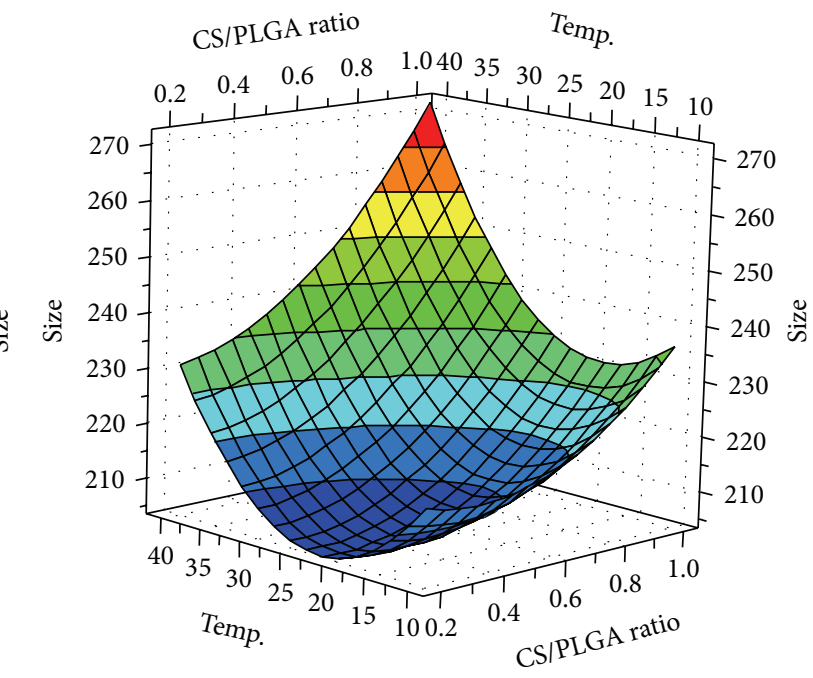

(b)

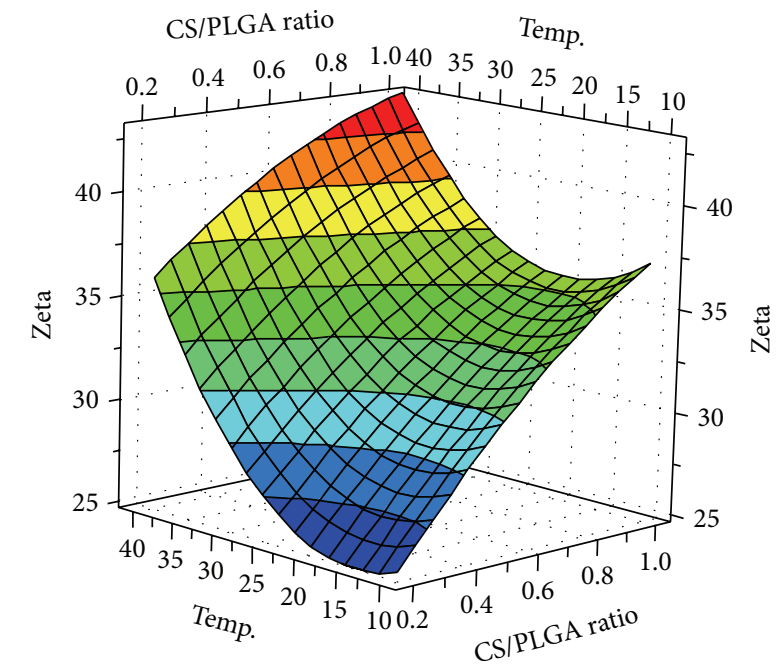

(d)

FIGURE 1: 4D response surface graphs of size in various conditions: at temperature $=25^{\circ} \mathrm{C}(\mathrm{a})$, at $\mathrm{pH}$ of CS solution $=4(\mathrm{~b})$, and at CS/PLGA mass ratio $=0.6(\mathrm{c})$ and of zeta potential of ART/PLGA-CS NPs at $\mathrm{pH}$ of CS solution $=4(\mathrm{~d})$.

Similar to $R^{2}, Q^{2}$ also had higher value at size compared with other responses. In addition, the $p$ value of the final regression model for each response variable was less than 0.05 and $p$ values for tests of lack of fit were more than 0.05 for all response variables, except for $Y_{2}$ (PDI). Thus, the results of two responses (excluding $Y_{2}$ ) were fitted to the quadratic models. The regression coefficients $\left(b_{i}\right)$ for each term in the full regression model were calculated by this software and performed in $(4)$ as below (only $b_{i}$ with $p$ values $<0.05$ ). From those data, it was clear that both size $\left(Y_{1}\right)$ and zeta potential $\left(Y_{3}\right)$ were affected by CS-to-PLGA mass ratio $\left(X_{1}\right)$, $\mathrm{pH}$ of CS solution $\left(X_{2}\right)$, and experimental temperature $\left(X_{3}\right)$ (all $p$ values of $b_{1}, b_{2}, b_{3}<0.05$ ). Furthermore, $Y_{1}$ and $Y_{3}$ were affected by quadratic term of $X_{2}$ ( $\mathrm{pH}$ of CS solution), as well as the interaction between CS-to-PLGA mass ratio $\left(X_{1}\right)$ and $\mathrm{pH}$ of CS solution ( $p$ values of $b_{5}, b_{7}<0.05$ ).
Since no pattern of models was found fitted for the output variable PDI, therefore, only two responses (size, zeta potential) were chosen for statistical modeling.

The equations fitted to the data were presented as follows:

$$
\begin{aligned}
Y_{1}= & 206.54+11.33 X_{1}+26.75 X_{2}+7.37 X_{3} \\
& +17.97 X_{2} X_{2}+12.95 X_{3} X_{3}-10.95 X_{1} X_{2} \\
Y_{3}= & 31.78+4.66 X_{1}-4.44 X_{2}+3.22 X_{3}+4.69 X_{2} X_{2} \\
& -4.43 X_{1} X_{2} .
\end{aligned}
$$

In addition, $4 \mathrm{D}$ response surface analysis also shows the influence of the input variables on the output variables. As shown in Figure 1(a), increasing in CS/PLGA ratio from 
TABLE 3: The optimal formulation of ART/PLGA-CS NPs.

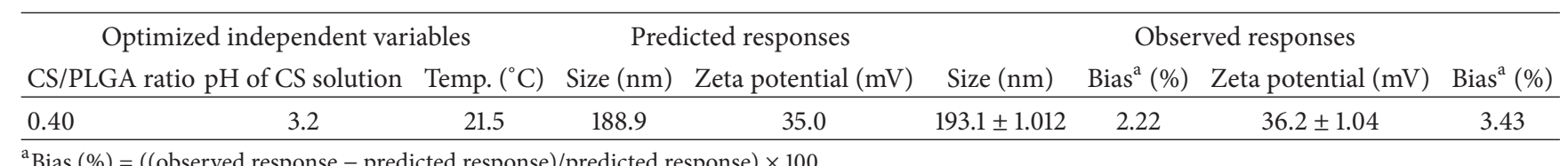

${ }^{\mathrm{a}} \operatorname{Bias}(\%)=(($ observed response - predicted response $) /$ predicted response $) \times 100$.

0.2 to $1.0(\mathrm{w} / \mathrm{w})$ significantly increased the size of ARTloaded PLGA-CS NPs. Particularly, a decrease in $\mathrm{pH}$ of CS solution led to the remarkable reduction of size of NPs. A reduction in $\mathrm{pH}$ leads to more ionization of the amino groups and hence more enhanced interaction probability with carboxylic end-groups of PLGA and therefore reduces particle diameter due to more convoluted chains of CS [12, 20]. When the experimental temperature was high (more than $25^{\circ} \mathrm{C}$ ), the increase in CS/PLGA ratio and $\mathrm{pH}$ of CS solution made the size of NPs rapidly increase (Figures $1(\mathrm{~b})$ and $1(\mathrm{c})$ ). The same relationships between the particle size and $\mathrm{pH}$ or temperature were obtained in the previous works about the CS NPs fabricated by ionic gelation technique $[21,22]$. Figure 1(d) shows that zeta potential of NPs was increased when there was an increase in CS/PLGA ratio or temperature, which could be explained by the increase in size of NPs. The mechanism of this adsorption maybe includes electrostatic attraction between positively charged CS and negatively charged PLGA surface to form the first monomolecular adsorption layer and hydrogen bonds ( $\mathrm{N}-$ $\mathrm{H})$, hydrophobic interactions, or Van der Waal's forces for subsequent layers [20].

The optimization of physicochemical properties of NPs was performed based on statistical analysis of experimental data. For constraints of responses, it is believed that "enhanced permeation and retention" (EPR) effect, which helps nanoparticles penetrate into solid tumors, is improved if size of particles is less than $200 \mathrm{~nm}$ [23]. About zeta potential, it was reported that a value below $-30 \mathrm{mV}$ (or above $+30 \mathrm{mV}$ ) indicates a stable colloidal dispersion [21, 24].

D-optimal designs are computer generated designs made for a specific problem and great flexibility in the specifications of the problem, particularly useful in the situation for inclusion of existing experiments and in optimization when we want to create an "odd" design [25]. According to our criteria for lower particle size and targeted zeta potential, Doptimal RSM in Modde software was used for optimization to obtain the final formulation with the optimized CS-to-PLGA mass ratio, $\mathrm{pH}$ of $\mathrm{CS}$ solution, and experimental temperature (Table 3). For model validation, PLGA-CS NPs were experimentally prepared and characterized following the optimized inputs $(n=3)$. As shown in Table 3 , all the bias was below $5 \%$ for each respective response, indicating the validity of generated models with no statistically significant difference and good correlation between predicted and experimental values [12].

\subsection{Physicochemical Characterization}

3.2.1. Particle Size and Morphology. Transmission electron microscopy images of ART/PLGA-CS NPs obtained from the optimal formulation are shown in Figure 2(a). The nanoparticles are spherical in shape and polydispersed with sizes in range of $190 \mathrm{~nm}$ which is consistent with the DLS data. In addition, EE and LC of these CS-coated NPs were maintained at high level with $77.30 \%$ and $19.97 \%$, respectively, during the coating step, similar to those of PLGA NPs as mentioned above [8].

3.2.2. FT-IR Analysis. FT-IR spectroscopy of ART, CS, PLGA, and ART-loaded PLGA-CS NPs was performed to demonstrate the presence of CS coating on PLGA nanoparticles (Figure 2(b)). The obtained FT-IR curve of ART/PLGA-CS NPs is similar to that of pure drug and excipients. From the FT-IR curve of CS, the intense peaks at 1646 and $1588 \mathrm{~cm}^{-1}$ confirm the presence of amide I and amide II in the chemical structure of chitosan. The chitosan's peak at $3355 \mathrm{~cm}^{-1}$ corresponds to the $\mathrm{N}-\mathrm{H}$ stretch which is overlapped with the $\mathrm{O}-\mathrm{H}$ stretch [26]. From the curve ART-PLGA-CHI in Figure 2(b), there are some peaks such as $\mathrm{COO}$ at $1747 \mathrm{~cm}^{-1}, \mathrm{CH}, \mathrm{CH}_{2}$, and $\mathrm{CH}_{3}$ stretching vibrations between 2945 and $2996 \mathrm{~cm}^{-1}$, overlapped peak of $\mathrm{N}-\mathrm{H}$ stretch, or $\mathrm{O}-\mathrm{H}$ stretch in the range of $3386-3485 \mathrm{~cm}^{-1}$, which are the same as those on curve ART, PLGA, or chitosan. In addition, the characteristic peaks of the amine groups $\left(\mathrm{NH}_{2}\right.$ bending vibration) of $\mathrm{CS}$ were observed at 1645 and $1616 \mathrm{~cm}^{-1}$, which are similar to those on curve chitosan. This confirmed the presence of CS onto the PLGA nanoparticle surface [9].

3.2.3. In Vitro Drug Release. Figure 2(c) shows the cumulative release curves of ART from the NPs. After 72 hours, about 64-74\% of the loaded ART in CS-uncoated and CS-coated NPs was released. All of the profiles were characterized by a period of rapid initial drug release followed by a continuous period of slow release after 24 hours due to diffusion or erosion of the PLGA matrix [27]. Compared with the PLGA NPs, the CS-coated NPs reduced the initial burst release during the first $24 \mathrm{~h}$. In detail, the cumulative release percentages of ART from the unmodified PLGA and CS-coated PLGA NPs were 44.61 and $21.02 \%$, respectively, at $2 \mathrm{~h}$, and 62.73 and $53.59 \%$, at $24 \mathrm{~h}$. This implied that the modification of the surface with CS had effectively changed the location of ART on the surface of the PLGA NPs, considered as a physical barrier, 


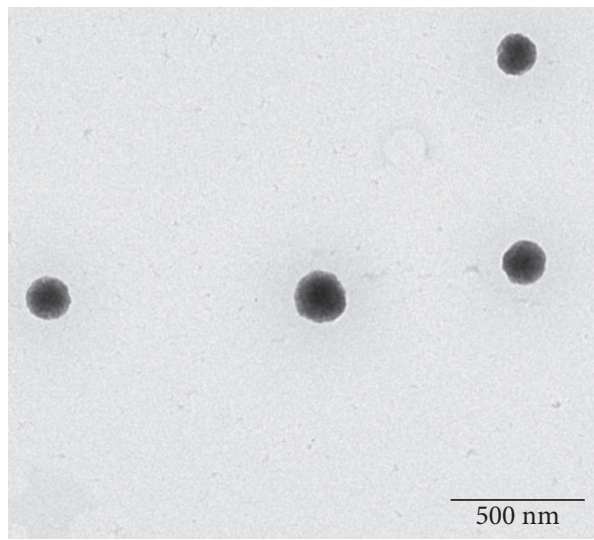

(a)

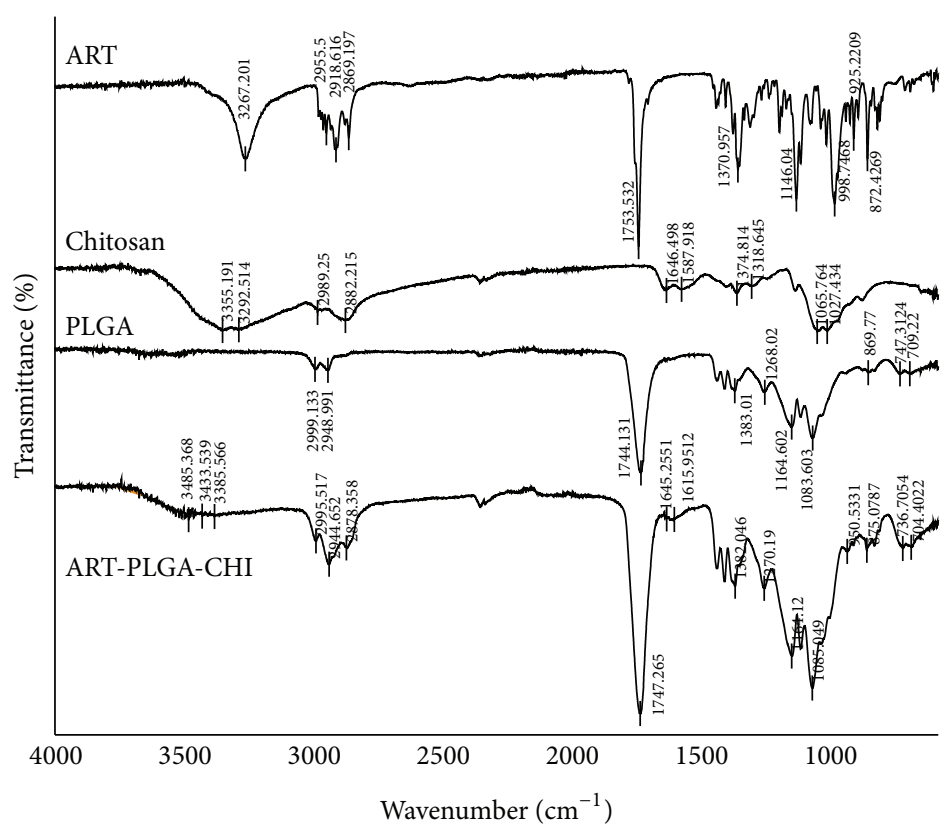

(b)

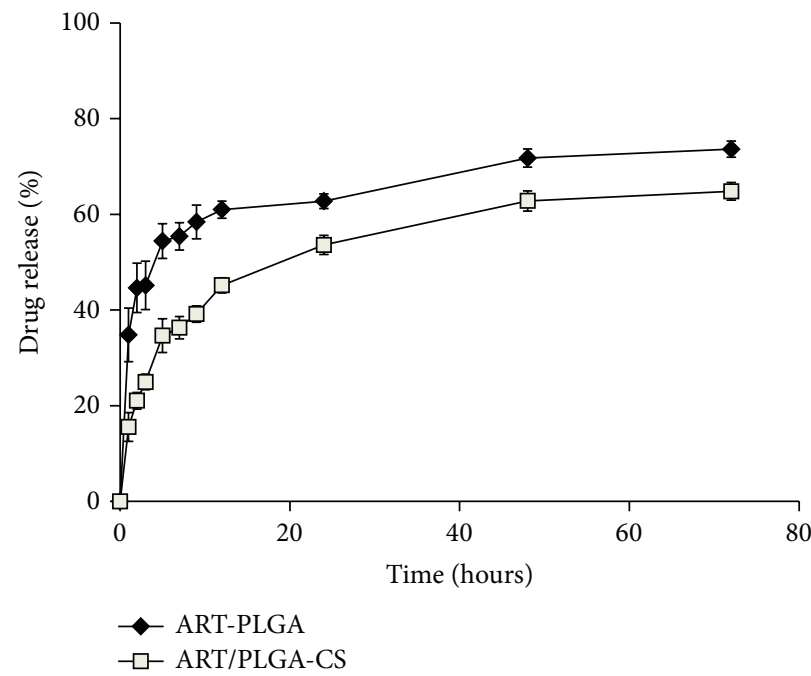

(c)

FIgURE 2: Physicochemical characterization: TEM image of ART/PLGA-CS NPs (Bar $=500 \mathrm{~nm})(\mathrm{a})$; FT-IR spectra of ART, CS, PLGA, and ART/PLGA-CS NPs (b); in vitro drug release profile of ART from ART/PLGA and ART/PLGA-CS NPs (c).

and consequently reduced the leaking of ART from the NPs. In addition, this slower release could be attributed to the ionic interaction of ART and CS coating layer [27-29]. The control release pattern of ART from PLGA-CS NPs was expected to release their payloads at targeted organelles so that the carrier will be safe on normal cells as well as expressing the highest activity on cancer cells.

3.3. Freeze-Thaw Study. Many components of the nanoparticle formulation have the crucial effect on the resistance of NPs to the different stresses during freeze-drying such as the type and concentration of cryoprotectants and the chemical groups attached to the NP surface [30]. Freeze-thaw is the simple procedure often used to rapidly evaluate how effective different types of cryoprotectants are before freeze-drying process [11]. During freezing process, the cryoprotectants form a glassy coating around the NPs protecting them against stresses like mechanical stress of ice crystals, thereby preventing aggregation $[11,31]$. The sugars which were used for this freeze-thaw study are the most popular cryoprotectants encountered in the literature for freeze-drying NPs. From Figure 3(a), particle size by using dextrose and sucrose was considered unchangeable compared with that by using trehalose, mannitol. The highest $S_{f} / S_{i}$ ratio was obtained 


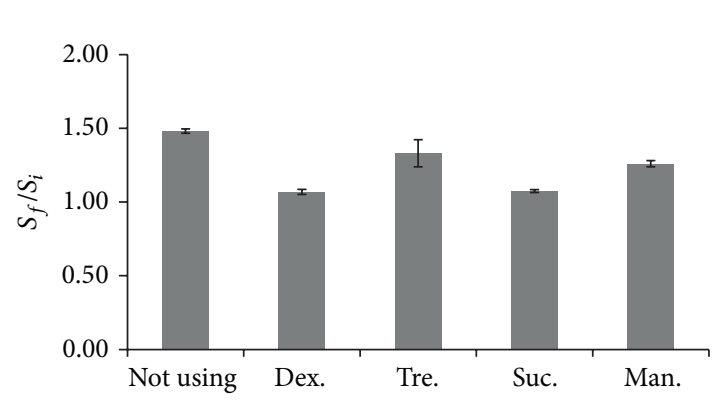

(a)

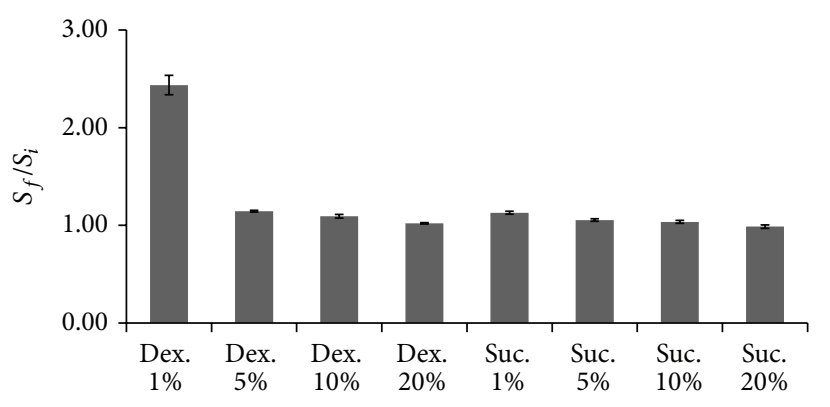

(b)

FIGURE 3: Effect of type of cryoprotectants on size of ART/PLGA-CS NPs after freeze-thaw study (a), effect of different concentrations of dextrose and sucrose on the size of ART/PLGA-CS nanoparticles during freeze-drying (b). (Dex.: dextrose anhydrous, Tre.: D-(+)-trehalose dihydrate, Suc.: sucrose, Man.: mannitol).

when trehalose was employed, which is approximately similar to that if no sugar was used $(1.330 \pm 0.091$ and $1.481 \pm 0.015$, resp.). As a result, dextrose and sucrose were chosen to use in freeze-drying process at various concentrations from $5 \%$ to $20 \%(\mathrm{w} / \mathrm{v})$ to evaluate their effectiveness of cryoprotection.

3.4. Lyophilization. The result in Figure $3(\mathrm{~b})$ shows that the cryoprotection of two carbohydrates was concentrationdependent. At low concentration of dextrose (below 10\%), there was some aggregation perhaps due to its insufficient concentration to form completely glassy coating outside of NPs [11]. The $S_{f} / S_{i}$ values were positively changed when the concentration of sucrose was increased from $5 \%$ to $20 \%$, but with insignificant decrease of only 0.065 . Sucrose is more effective on preventing particle aggregation than dextrose at the same concentration; for example, $S_{f} / S_{i}$ ratios for $10 \%$ of dextrose and sucrose were $1.092 \pm 0.019$ and $1.035 \pm 0.016$, respectively. According to an excellent review of Abdelwahed et al. [30], it has been previously found that disaccharides like sucrose were more effective to preserve size of griseofulvin loaded NPs during freeze-drying than monosaccharides like dextrose in this study, perhaps due to the amorphous state of sucrose-containing NPs compared to the crystal state of dextrose-containing NPs. Some hypotheses were proposed to explain this difference among them including the particle isolation hypothesis and the water replacement hypothesis [30]. Consequently, $5 \%$ of sucrose was chosen for the next lyophilization.

3.5. Intracellular Uptake by FACs. In order to rule out the possibility of enhanced adhesion of nanoparticles to the cellular surface by CS modification and improved intracellular uptake of PLGA-CS into the MCF-7 and A549 cells, flow cytometry was further used to quantitatively determine. As shown in Figure 4, the CS-coated PLGA NPs exhibited higher levels of cellular uptake (the fluorescence slightly shifted towards the right) compared with the uncoated PLGA NPs. The C6loaded PLGA-CS NPs clearly exhibited enhanced cellular uptake levels in both dose-dependent and time-dependent manner. The lower cellular uptake level of the uncoated PLGA
NPs was attributed to their negative charge, which would have been repelled by the negatively charged cell surface $[10,17]$. These results suggested that, due to positive charge, CS modification could enhance cellular uptake levels, leading to higher apoptosis of MCF-7 and A549 cells [32].

3.6. In Vitro Cytotoxicity Test. We studied the inhibitory effect of ART-loaded NPs on the proliferation of MCF-7 and A549 cells using an MTT assay after $24 \mathrm{~h}$ treatment with the nanoparticle formulations at the equivalent ART doses of $6.25,12.5,25,50$, and $100 \mu \mathrm{g} / \mathrm{mL}$, respectively. Figure 5 clearly showed that blank CS-coated NPs had no significant influence on the cell viability of MCF-7 and A549 cells at $24 \mathrm{~h}$ after administration (more than $80 \%$ ), which means that these blank NPs were not cytotoxic in the concentration range of interest [15]. In case of free ART, ART-loaded NPs with the above concentration, the growth of the MCF-7 and A549 cells were significantly inhibited in a dose-dependent manner. Compared with free ART, the cell viability of the MCF-7 and A549 cells was much reduced when treated with the ART-loaded NP formulations $(p<0.05)$, suggesting that the ART-loaded NPs delivered a higher level of cytotoxicity and especially showed significant toxicity to A549 cells. In the current study, it was found that the CS-surface modified PLGA NPs could significantly enhance the cellular uptake of ART in MCF-7 and A549 cells (Figure 6) and therefore exhibited higher levels of cytotoxicity towards these cancer cells compared with ART-loaded PLGA NPs $(p<0.05)$. These higher levels of cytotoxicity were attributed to the effect of the NPs carrying the drug into the cells via endocytosis and the increased cationic nature of CS-decorated NPs around a relatively acidic $\mathrm{pH}$ environment of the cancer cells compared to neutral $\mathrm{pH}$ of normal cells $[19,33]$. These results suggested that the use of CS-surface modified PLGA NPs as a carrier for ART could be promising for better effects on cancer cells [10].

3.7. Nuclei Morphology Characterization. Hoechst 33342, a cell-permeable fluorescent compound that is able to stain DNA by binding with high affinity to the minor groove of AT-rich DNA sequence, was used to assess changes in nuclear 


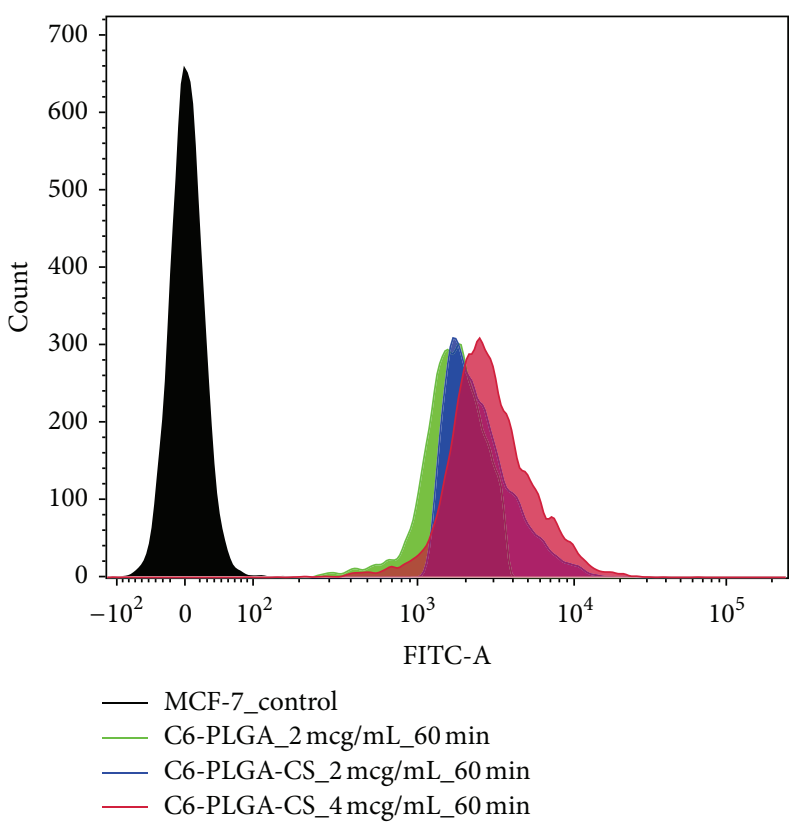

(a)

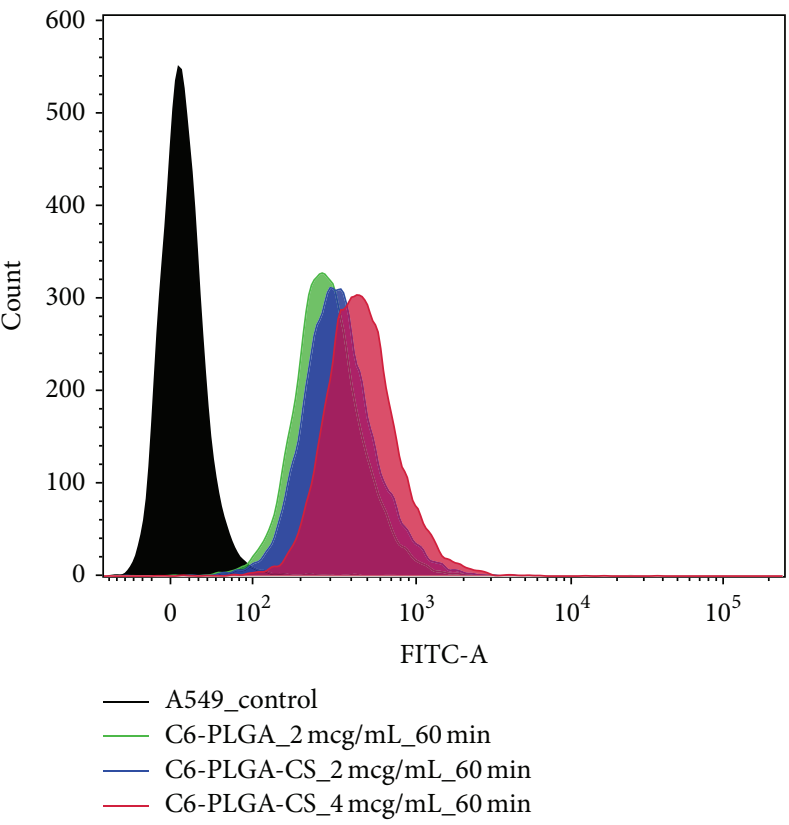

(c)

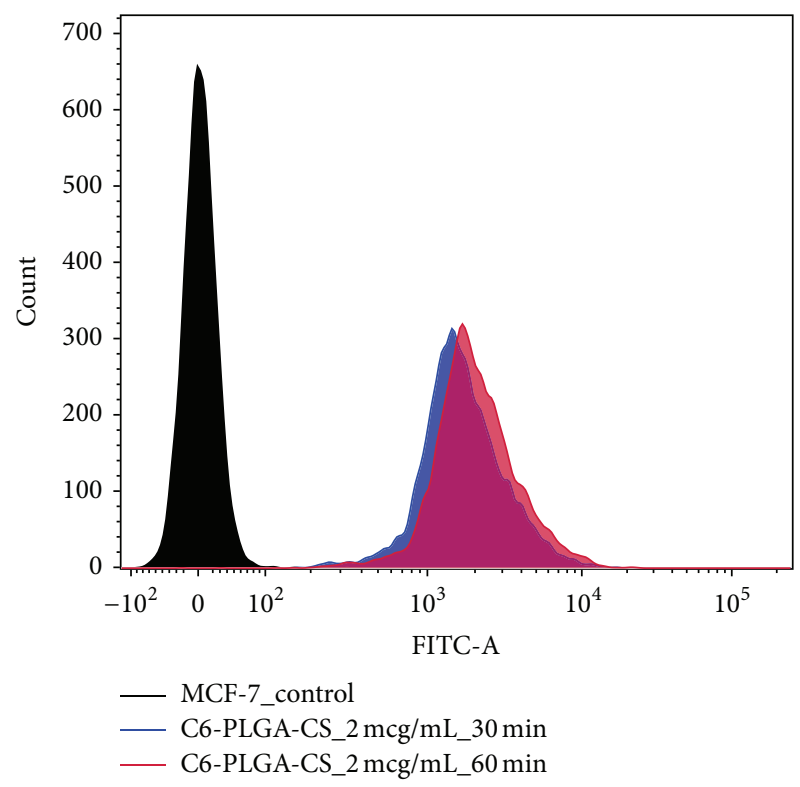

(b)

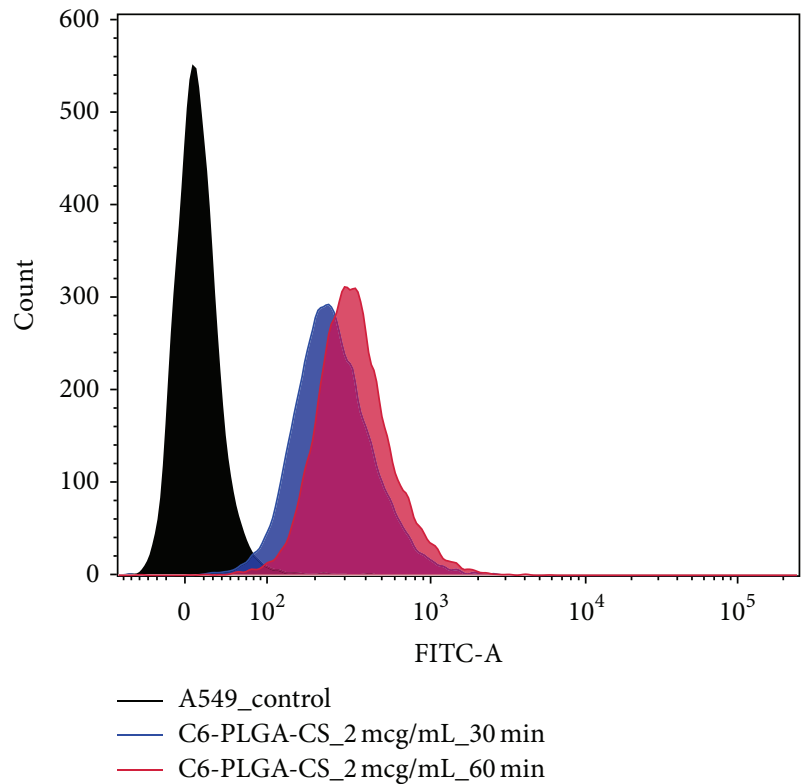

(d)

Figure 4: Intracellular uptake of C6-PLGA and C6-PLGA-CS by flow cytometry analysis on MCF-7 (a, b) and A549 (c, d).

morphology of MCF-7 and A549 cells following ART treatment. The nuclei in untreated cells (control group) exhibited diffused staining of the chromatin (Figure 6). However, after exposure to free ART, PLGA NPs, and CS-coated PLGA NPs at the dose of $50 \mu \mathrm{g} / \mathrm{mL}$ ART for $24 \mathrm{~h}$, the cells underwent typical morphologic changes of apoptosis, perhaps such as cell shrinkage, nuclear collapse, without disruption in cell membrane (Figure 6) [34]. The fluorescent intensity in cells incubated with PLGA-CS NPs was higher than that with PLGA NPs and free ART. It is suggested that CS-coated NPs were more effective on enhancing the cell apoptosis than CS-uncoated NPs, which is similar to the previous report about DNA damage and cell death induced by ART via the apoptotic pathway $[32,35]$.

\section{Conclusions}

In summary, this study designed the optimum formulation of CS-modified PLGA NPs by using MODDE software. The analysis of surface charge by zeta potential and surface 


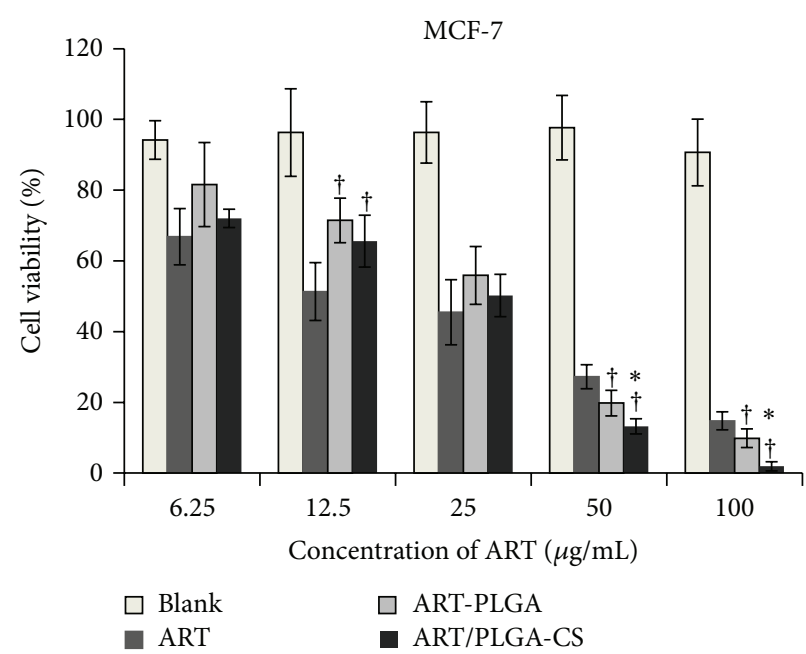

(a)

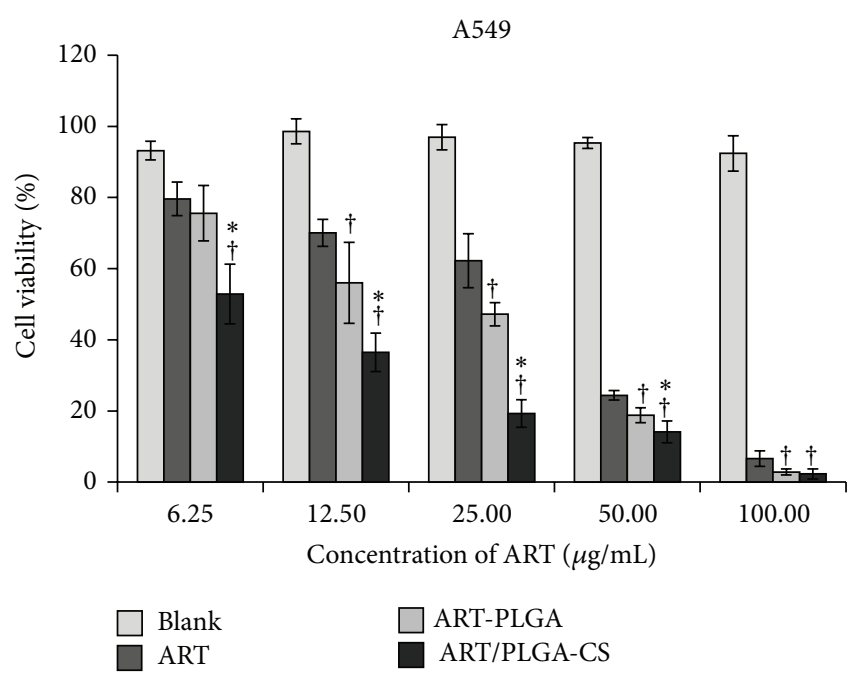

(b)

FIGURE 5: In vitro cytotoxicity of blank PLGA-CS, free ART, ART-PLGA NPs, and ART/PLGA-CS NPs on MCF-7 and A549 cells after $24 \mathrm{~h}$ exposure. “†”: free ART versus NPs, $p<0.05$; “*”: CS-coated NPs versus CS-uncoated NPs, $p<0.05$.
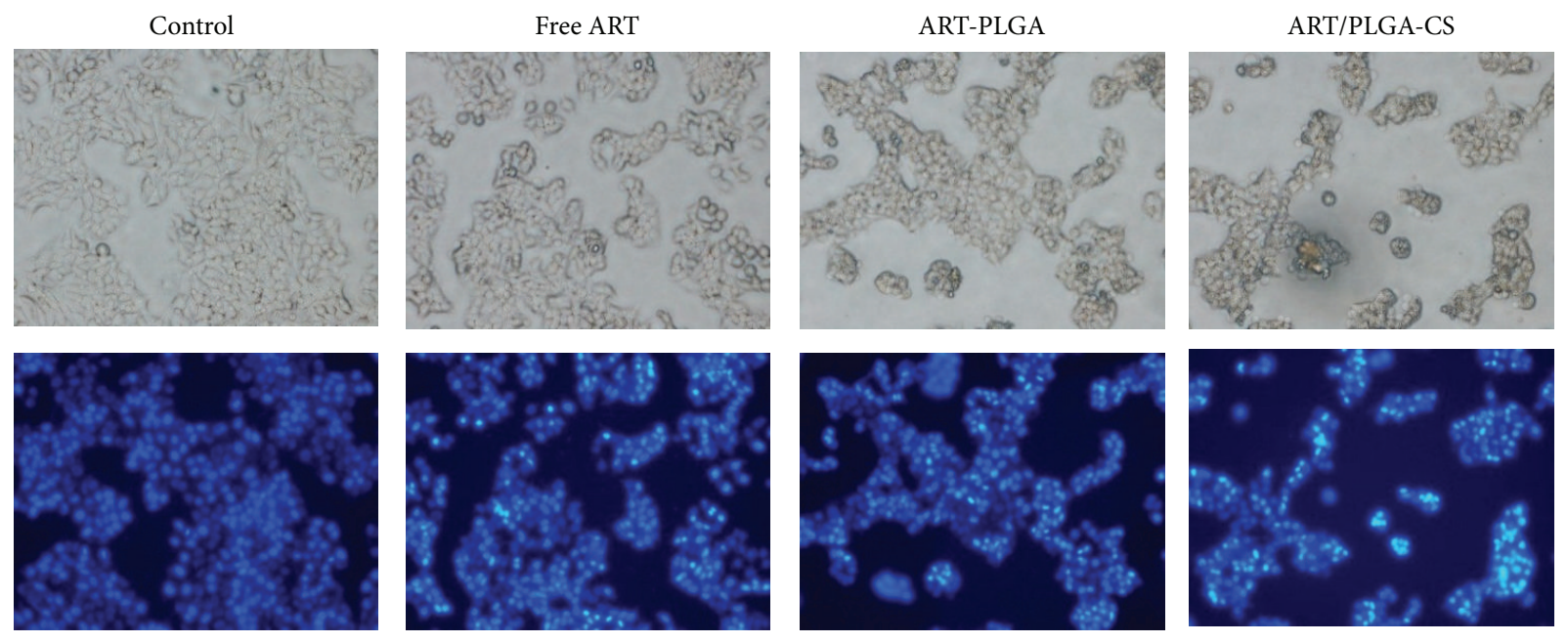

(a)
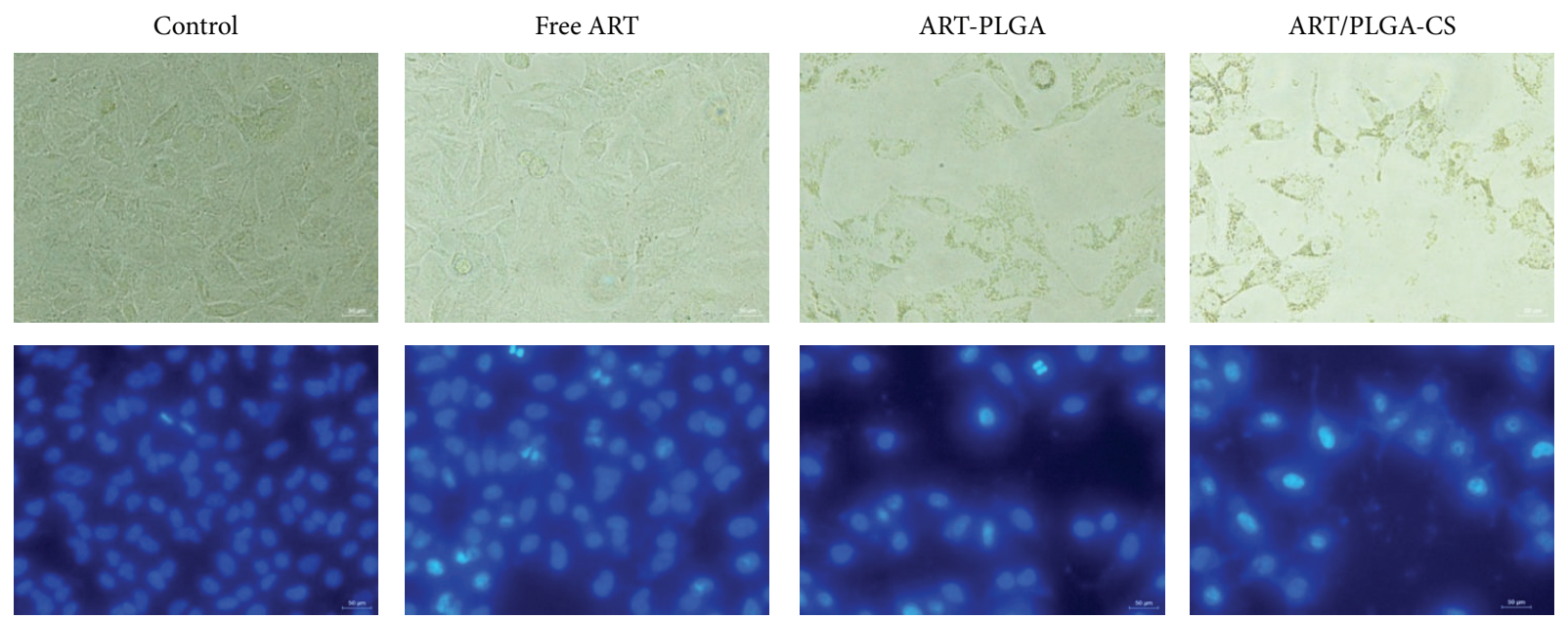

(b)

FIGURE 6: Nuclei morphology characterization using confocal laser scan microscopy after treatment for 24h with free ART, ART/PLGA, and ART/PLGA-CS NPs on MCF-7 (a) and A549 cells (b). 
composition by FT-IR spectroscopy proved the presence of CS on surface of PLGA NPs. This formulation can reduce the burst initial drug release compared with uncoated NPs. In addition, the better uptake of CS-coated PLGA NPs may contribute to improving in vitro cytotoxicity which induced apoptosis pathway on MCF-7, A549 cancer cell lines.

\section{Conflict of Interests}

The authors declare that there is no conflict of interests regarding the publication of this paper.

\section{Acknowledgment}

This research is funded by the Vietnam National Foundation for Science and Technology Development (NAFOSTED) under Grant no. 106-YS.05-2013.15.

\section{References}

[1] A. Hamacher-Brady, H. A. Stein, S. Turschner et al., "Artesunate activates mitochondrial apoptosis in breast cancer cells via ironcatalyzed lysosomal reactive oxygen species production," The Journal of Biological Chemistry, vol. 286, no. 8, pp. 6587-6601, 2011.

[2] M. P. Crespo-Ortiz and M. Q. Wei, "Antitumor activity of artemisinin and its derivatives: from a well-known antimalarial agent to a potential anticancer drug," Journal of Biomedicine and Biotechnology, vol. 2012, Article ID 247597, 18 pages, 2012.

[3] T. Efferth, A. Sauerbrey, A. Olbrich et al., "Molecular modes of action of artesunate in tumor cell lines," Molecular Pharmacology, vol. 64, no. 2, pp. 382-394, 2003.

[4] K. T. Batty, K. F. Ilett, M. E. Davis, and Timothy, "Chemical stability of artesunate injection and proposal for its administration by intravenous infusion," Journal of Pharmacy and Pharmacology, vol. 48, no. 1, pp. 22-26, 1996.

[5] N. Durán, P. D. Marcato, Z. Teixeira, M. Durán, F. T. M. Costa, and M. Brocchi, "State of the art of nanobiotechnology applications in neglected diseases," Current Nanoscience, vol. 5, no. 4, pp. 396-408, 2009.

[6] F. Danhier, E. Ansorena, J. M. Silva, R. Coco, A. Le Breton, and V. Préat, "PLGA-based nanoparticles: an overview of biomedical applications," Journal of Controlled Release, vol. 161, no. 2, pp. 505-522, 2012.

[7] H. K. Makadia and S. J. Siegel, "Poly Lactic-co-Glycolic Acid (PLGA) as biodegradable controlled drug delivery carrier," Polymers, vol. 3, no. 3, pp. 1377-1397, 2011.

[8] H. T. Nguyen, T. H. Tran, J. O. Kim, C. S. Yong, and C. N. Nguyen, "Enhancing the in vitro anti-cancer efficacy of artesunate by loading into poly-D,L-lactide-co-glycolide (PLGA) nanoparticles," Archives of Pharmacal Research, vol. 38, no. 5, pp. 716-724, 2014.

[9] Y. Wang, P. Li, and L. Kong, "Chitosan-modified PLGA nanoparticles with versatile surface for improved drug delivery," AAPS PharmSciTech, vol. 14, no. 2, pp. 585-592, 2013.

[10] H.-L. Chen, Y.-X. Wang, P. Zhou et al., "Chitosan surfacemodified PLGA nanoparticles: preparation, characterization, and evaluation of their in vitro drug-release behaviors and cytotoxicities," Current Nanoscience, vol. 10, no. 2, pp. 255-262, 2014.
[11] P. V. Date, A. Samad, and P. V. Devarajan, "Freeze thaw: a simple approach for prediction of optimal cryoprotectant for freeze drying," AAPS PharmSciTech, vol. 11, no. 1, pp. 304-313, 2010.

[12] S. M. Abdel-Hafez, R. M. Hathout, and O. A. Sammour, "Towards better modeling of chitosan nanoparticles production: screening different factors and comparing two experimental designs," International Journal of Biological Macromolecules, vol. 64, pp. 334-340, 2014.

[13] D. C. Montgomery, Design and Analysis of Experiments, John Wiley \& Sons, 8th edition, 2012.

[14] C. Nguyen, J. M. Christensen, and T. Nguyen, "Application of Doptimal study design with contour surface response for designing sustained release gliclazide matrix tablets," Pharmacology \& Pharmacy, vol. 5, no. 7, pp. 620-635, 2014.

[15] T. H. Tran, T. Ramasamy, H. J. Cho et al., "Formulation and optimization of raloxifene-loaded solid lipid nanoparticles to enhance oral bioavailability," Journal of Nanoscience and Nanotechnology, vol. 14, no. 7, pp. 4820-4831, 2014.

[16] T. Ramasamy, T. H. Tran, H. J. Cho et al., "Chitosan-based polyelectrolyte complexes as potential nanoparticulate carriers: physicochemical and biological characterization," Pharmaceutical Research, vol. 31, no. 5, pp. 1302-1314, 2014.

[17] M. Wang, Y. Zhang, J. Feng et al., "Preparation, characterization, and in vitro and in vivo investigation of chitosan-coated poly (d,l-lactide-co-glycolide) nanoparticles for intestinal delivery of exendin-4," International Journal of Nanomedicine, vol. 8, pp. 1141-1154, 2013.

[18] H. Meng, K. Xu, Y. Xu et al., "Nanocapsules based on mPEGylated artesunate prodrug and its cytotoxicity," Colloids and Surfaces B: Biointerfaces, vol. 115, pp. 164-169, 2014.

[19] R. Yang, W.-S. Shim, F.-D. Cui et al., "Enhanced electrostatic interaction between chitosan-modified PLGA nanoparticle and tumor," International Journal of Pharmaceutics, vol. 371, no. 1-2, pp. 142-147, 2009.

[20] C. Guo and R. A. Gemeinhart, "Understanding the adsorption mechanism of chitosan onto poly(lactide-co-glycolide) particles," European Journal of Pharmaceutics and Biopharmaceutics, vol. 70, no. 2, pp. 597-604, 2008.

[21] C. Bulmer, A. Margaritis, and A. Xenocostas, "Production and characterization of novel chitosan nanoparticles for controlled release of rHu-erythropoietin," Biochemical Engineering Journal, vol. 68, pp. 61-69, 2012.

[22] W. Fan, W. Yan, Z. Xu, and H. Ni, "Formation mechanism of monodisperse, low molecular weight chitosan nanoparticles by ionic gelation technique," Colloids and Surfaces B: Biointerfaces, vol. 90, no. 1, pp. 21-27, 2012.

[23] G. Mattheolabakis, B. Rigas, and P. P. Constantinides, "Nanodelivery strategies in cancer chemotherapy: biological rationale and pharmaceutical perspectives," Nanomedicine, vol. 7, no. 10, pp. 1577-1590, 2012.

[24] Y.-Z. Du, L. Wang, H. Yuan, X.-H. Wei, and F.-Q. Hu, "Preparation and characteristics of linoleic acid-grafted chitosan oligosaccharide micelles as a carrier for doxorubicin," Colloids and Surfaces B: Biointerfaces, vol. 69, no. 2, pp. 257-263, 2009.

[25] L. Eriksson, E. Johansson, N. Kettaneh-Wold, C. Wikström, and S. Wold, Design of Experiments: Principles and Applications, Umetrics, Umeå Learnways AB, Stockholm, Sweden, 2000.

[26] P. Mukhopadhyay, K. Sarkar, S. Bhattacharya, A. Bhattacharyya, R. Mishra, and P. P. Kundu, "pH sensitive $N$-succinyl chitosan grafted polyacrylamide hydrogel for oral insulin delivery," Carbohydrate Polymers, vol. 112, pp. 627-637, 2014. 
[27] K. Tahara, H. Yamamoto, N. Hirashima, and Y. Kawashima, "Chitosan-modified poly(d,l-lactide-co-glycolide) nanospheres for improving siRNA delivery and gene-silencing effects," European Journal of Pharmaceutics and Biopharmaceutics, vol. 74, no. 3, pp. 421-426, 2010.

[28] C. Lee, J. S. Choi, I. Kim et al., "Long-acting inhalable chitosancoated poly(lactic-co-glycolic acid) nanoparticles containing hydrophobically modified exendin- 4 for treating type 2 diabetes," International Journal of Nanomedicine, vol. 8, pp. 29752983, 2013.

[29] K. Zhao, Y. Zhang, X. Zhang et al., "Chitosan-coated poly(lacticco-glycolic) acid nanoparticles as an efficient delivery system for Newcastle disease virus DNA vaccine," International Journal of Nanomedicine, vol. 9, no. 1, pp. 4609-4619, 2014.

[30] W. Abdelwahed, G. Degobert, S. Stainmesse, and H. Fessi, "Freeze-drying of nanoparticles: formulation, process and storage considerations," Advanced Drug Delivery Reviews, vol. 58, no. 15, pp. 1688-1713, 2006.

[31] S. Bozdag, K. Dillen, J. Vandervoort, and A. Ludwig, “The effect of freeze-drying with different cryoprotectants and gammairradiation sterilization on the characteristics of ciprofloxacin HCl-loaded poly(D,L-lactide-glycolide) nanoparticles," Journal of Pharmacy and Pharmacology, vol. 57, no. 6, pp. 699-707, 2005.

[32] T. H. Tran, T. D. Nguyen, B. K. Poudel et al., "Development and evaluation of artesunate-loaded chitosan-coated lipid nanocapsule as a potential drug delivery system against breast cancer," AAPS PharmSciTech, 2015.

[33] Y.-I. Chung, J. C. Kim, Y. H. Kim et al., "The effect of surface functionalization of PLGA nanoparticles by heparinor chitosan-conjugated Pluronic on tumor targeting," Journal of Controlled Release, vol. 143, no. 3, pp. 374-382, 2010.

[34] Y.-Y. Lu, T.-S. Chen, J.-L. Qu, W.-L. Pan, L. Sun, and X.-B. Wei, "Dihydroartemisinin (DHA) induces caspase-3dependent apoptosis in human lung adenocarcinoma ASTC-a-1 cells," Journal of Biomedical Science, vol. 16, article 16, 2009.

[35] P. C. H. Li, E. Lam, W. P. Roos, M. Z. Zdzienicka, B. Kaina, and T. Efferth, "Artesunate derived from traditional Chinese medicine induces DNA damage and repair," Cancer Research, vol. 68, no. 11, pp. 4347-4351, 2008. 

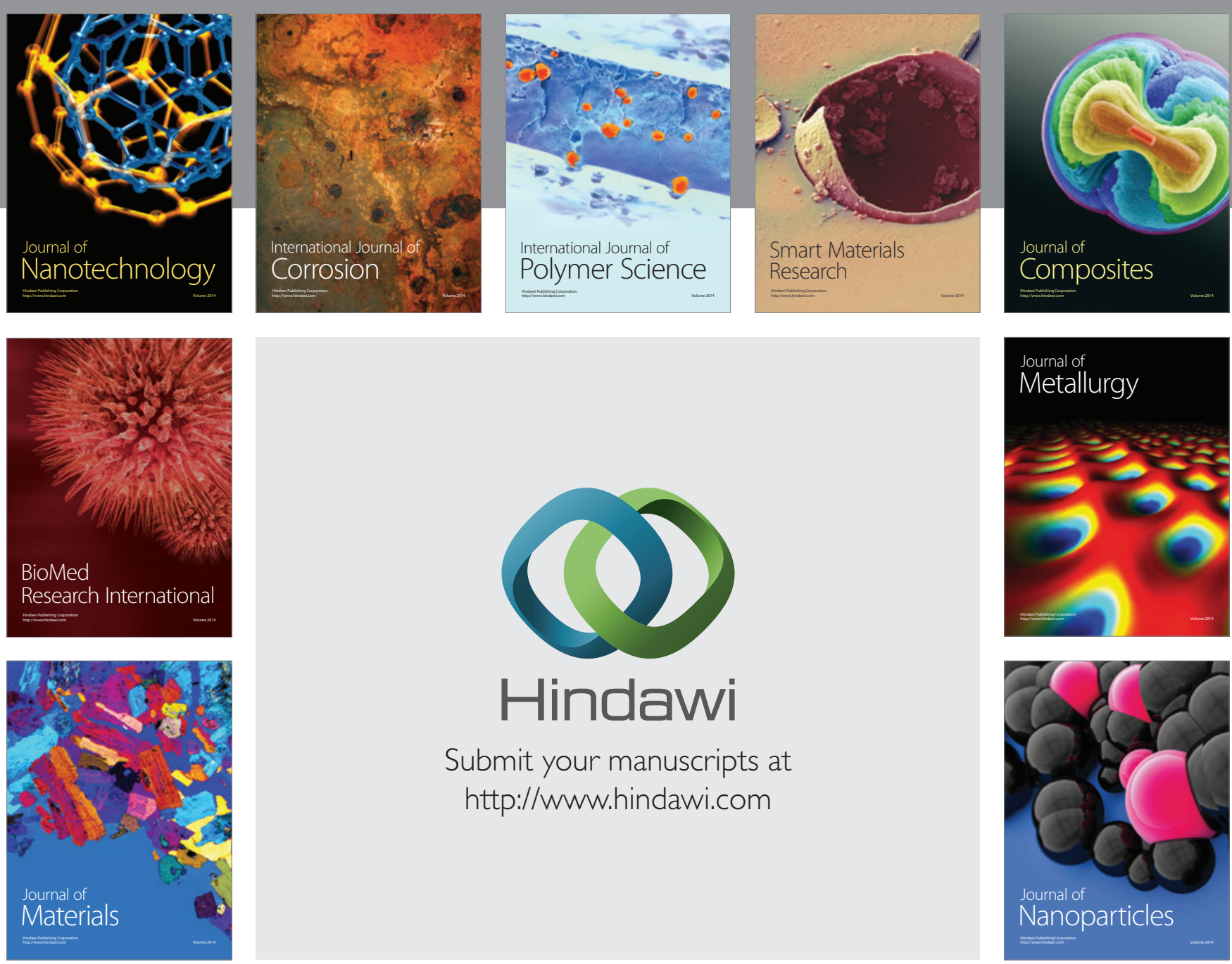

Submit your manuscripts at http://www.hindawi.com
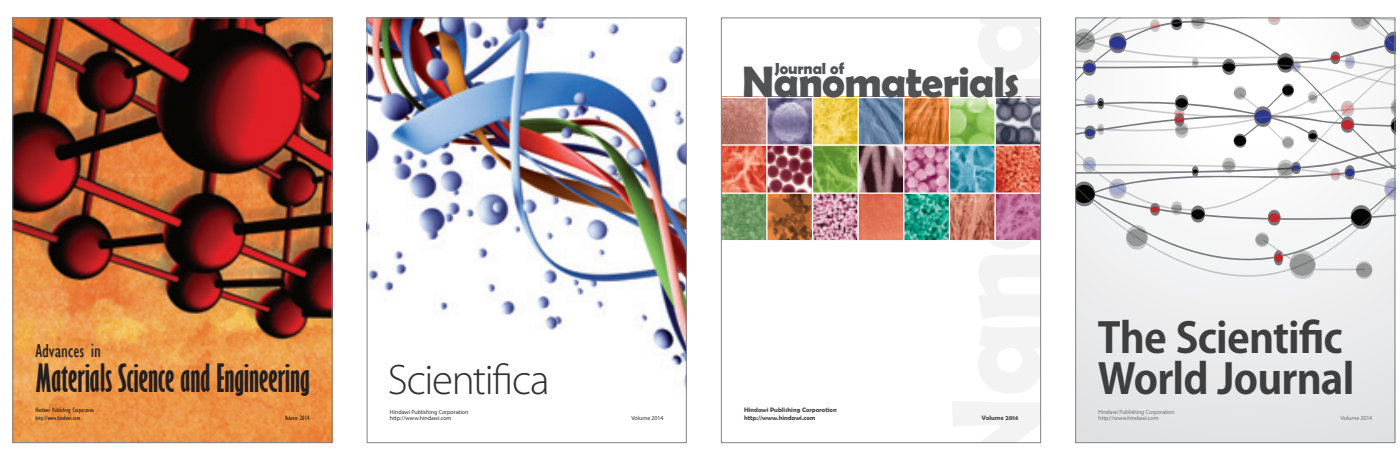

\section{The Scientific World Journal}
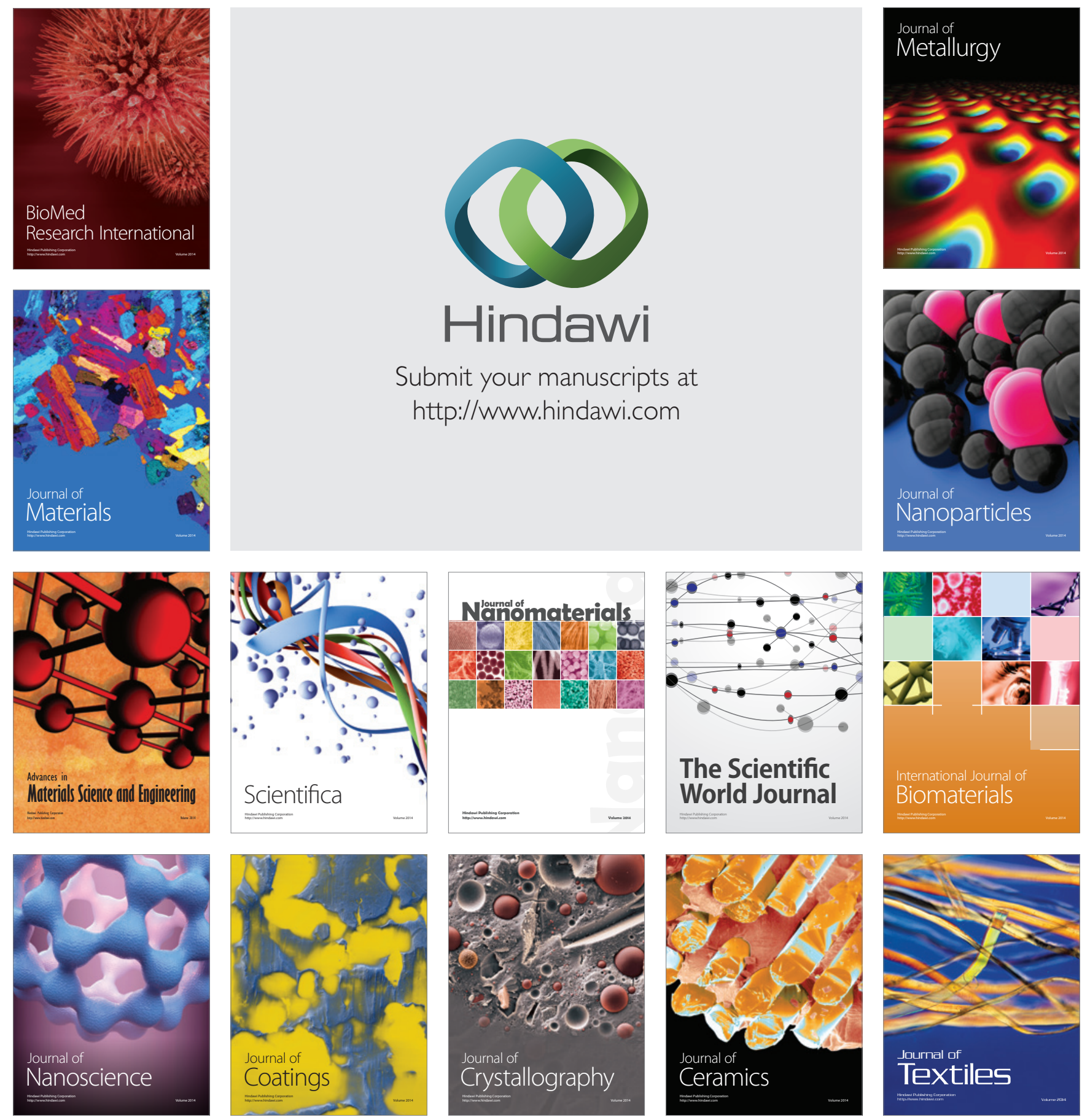